Note de lecture, Mosaïque, Gérer et Comprendre

\title{
Mais où sont les neiges d'antan ?
}

A propos du livre de Geoffroy de Lagasnerie, Logique de la création, coll. Histoire de la pensée, Paris, Fayard, 2011

Jean-Marc WELLER

A l'heure où la réforme des mondes de la recherche et de l'université ne jurent que par «l'excellence », où la qualité et l'autonomie n'ont jamais autant été portés au pinacle pour valoriser les milieux académiques et promouvoir ce qu'il y a de meilleur, de plus grand, de plus stratégique, de plus « excellent», le livre de Geoffroy de Lagasnerie ressemble fort à un ouvrage salutaire. Avant de tenter d'apprécier la portée de l'analyse - à propos de laquelle, disons-le d'emblée, nous demeurons partagé —, rappelons préalablement le projet de l'auteur: produire une critique de la forme actuelle de la production académique. Pour ce faire, Lagasnerie reconsidère l'histoire intellectuelle principalement des années 60 et 70, focalisant son attention sur les itinéraires des grands noms qui ont alors dominé les débats dans les sciences humaines et sociales, et ce pour poser une question : quelles sont les forces qui ont rendu possible l'émergence de cette intense créativité ? Pour le dire autrement, des pensées novatrices comme celles de Lévi-Strauss, Barthes, Derrida, Foucault, Deleuze ou Bourdieu, qui se sont toutes constituées à distance des normes académiques, seraient-elles encore possibles aujourd'hui, compte tenu des transformations actuelles du monde de la recherche?

L'hypothèse qui inspire l'ouvrage est éminemment sociologique : de la même manière que Durkheim considérait le suicide, non comme un acte exclusivement individuel, mais bien comme un phénomène social lié à une organisation de la société, Lagasnerie invite à considérer la création intellectuelle comme le produit d'un "penchant collectif», d'une capacité à fabriquer des individualités créatrices qui dépend d'un milieu, d'un contexte en mesure de rendre possible la production d'«espaces de liberté ». Dans cette perspective, l'auteur explore l'histoire intellectuelle de la période de l'après-guerre aux années 80 à travers trois chapitres, qui invitent respectivement à enquêter sur la nature de l' «attitude créatrice » (chapitre 1), à se demander ce qu'est un auteur (chapitre 2) et à s'interroger sur les conditions institutionnelles de cette production d'idées nouvelles (chapitre 3). A la lumière de 
l'enseignement fourni par cette histoire, l'auteur réexamine les transformations du monde de la recherche d'aujourd'hui, faisant écho aux ambiguïtés de la politique actuellement à l'œuvre, amplement posée en introduction et discutée en conclusion.

$\mathrm{Au}$ travers de cette relecture critique, l'auteur développe avec un indéniable brio plusieurs thèses qui inspirent, à des degrés divers, les différents chapitres du livre. Je propose d'en retenir trois.

Une première thèse considère que créer, c'est dévier. C'est produire de l'inédit, de la singularité, de la différence, qu'un auteur accomplit en s'écartant des normes établies. Toutes les innovations intellectuelles le montrent: elles procèdent par rupture. Ainsi, lorsque Lagasnerie examine attentivement l'itinéraire de Michel Foucault, il rappelle que ses premiers écrits s'inscrivent parfaitement dans la double tradition du marxisme et de phénoménologie, c'est-à-dire dans ce qui constituait, dans les années cinquante, les paradigmes intellectuels convenus du champ philosophique et politique de l'époque. Pour écrire l'Histoire de la folie à l'âge classique, il lui faudra rompre. C'est, d'ailleurs, ce même mouvement de discontinuité, révélant le dépassement des paradigmes institués, que décrivent les trajectoires d'un Bourdieu, d'un Derrida ou d'un Deleuze. La rupture, que la logique de la création impose d'accomplir, est en fait triple. Elle est d'abord personnelle : on rompt avec soi-même. Elle est également paradigmatique : on rompt avec une théorie établie, un horizon d'intelligibilité majoritaire. Enfin, elle est institutionnelle : on rompt avec les délimitations convenues d'un champ, d'une discipline. En bref, on rompt avec l'académisme. Il faut bien prendre la mesure de la chose. Lorsque Lagasnerie retrace l'itinéraire de Lévi-Strauss, il insiste sur l'hétérogénéité des milieux qui inspire son imagination où se côtoient pêle-mêle l'anthropologie, la révolution linguistique engagée par Saussure, l'ethnologie américaine découverte lors de son exil à New York, mais aussi le mouvement surréaliste auquel l'introduit André Breton rencontré sur le bateau pour l'Amérique. De même, la recherche d'un nouveau langage théorique pour explorer la maladie mentale, la psychologie et la folie doit, chez Foucault, à l'étude comparée des mythes et des religions posée par Dumézil, mais aussi à la puissance des œuvres littéraires de Bataille et Blanchot, voire à la musique contemporaine avec Boulez ou Barraqué. Car c'est cette intense circulation d'idées et de mondes qui fait le germe de l'exaltation intellectuelle, propice à la création. Bref, la logique de l'innovation - en tant qu'acte créatif — ne s'épuise pas dans la seule manière dont le 
champ académique s'est constitué, et que l'innovateur contribuerait, par rupture, à renouveler. Elle puise son énergie dans un ailleurs, un imaginaire foisonnant et multiple de références qui composent l'espace intérieur que le chercheur s'est lui-même constitué. C'est dans cette perspective que Lasganerie situe les auteurs auxquels il s'intéresse : des penseurs opposés à la posture académique, à ses spécialisations disciplinaires, à ses objets constitués. Il interroge, à l'aune de ce constat, les limites de l'actuelle politique qui, au nom d'une "professionnalisation» des sciences humaines, entend désormais réguler l'activité de recherche au prix d'une définition plus serrée de la «qualité » scientifique, supposant la quantification bibliométrique, le classement des revues et une plus grande fermeture des disciplines sur elles-mêmes. Ces critères ne risquent-ils pas de renforcer ce qui est convenu et établi, faisant passer pour un progrès ce qui ressemble, en vérité, à une régression ? L'histoire des idées ne montre t-elle pas, au contraire, qu'innover suppose de rompre avec la figure du chercheur intégré à sa discipline?

Une deuxième thèse rappelle qu'il n'y a pas de création sans espaces de liberté. Car si créer, c'est rompre, en faisant dialoguer des idées issues de domaines ou d'horizons différents et en dessinant des circulations inédites entre des mondes réputés distincts, encore faut-il que des lieux, des agencements permettent de telles hybridations. L'imagination féconde du chercheur est à ce prix. Or, aujourd'hui, dans l'Université, la normalisation disciplinaire a progressivement conduit à faire disparaître ces espaces qui, au cours des années 60 et 70, avaient émergé en rupture avec les normes académiques, véritables lieux mêmes de la production de cette circulation d'idées et de milieux hétérogènes. Ainsi, la VIe section de 1'Ecole Pratique des Hautes Etudes — haut siège de l'anti-disciplinarité - s'est transformé en un lieu parfaitement conventionnel, organisé en disciplines. Le centre expérimental de Vincennes, indifférent à l'origine aux titres scolaires et aux positions académiques, est redevenu une université comme les autres. Des revues comme Critique ou les Actes de la Recherche en Sciences Sociales se sont dotées de comités et de normes d'évaluation qui font disparaître l'hérésie et la création qui les caractérisaient. Si la «logique de la création» suppose l'existence d'authentiques espaces de liberté, lieux hybrides d'échanges et de confrontation, les transformations en cours ne contribuent t-elles pas, au nom de la normalisation disciplinaire de l'activité scientifique, a contrario, à leur extinction ?

Une troisième thèse pose, enfin, que créer, c'est s'adresser à un public. C'est produire des énoncés qui engage le chercheur en tant qu'auteur, et qui conduit Lagasnerie à souligner 
combien, de Sartre à Deleuze en passant par Foucault ou Bourdieu, les livres qu'ils ont écrits ont trouvé un lectorat qui dépasse de loin les seuls cercles académiques. A la question éminemment centrale de savoir «pour qui écrit-on ?», ils ont clairement élaboré des textes pour des publics hétérogènes et composés de «non spécialistes ». Lagasnerie rappelle combien Foucault espérait écrire « pour que cela serve », comment Bourdieu entendait publier pour « les dominés ». Car, qu'on le veuille ou non, les innovateurs emblématiques de $L a$ logique de la création demeurent des intellectuels politiquement engagés, ouverts sur le monde et les problèmes qui s'y posent, de sorte que si leurs travaux rencontrent un indéniable succès auprès de publics fort variés, c'est aussi en raison de leur stratégie d'écriture : sortir de l'académisme. Aujourd'hui, à l'Université, le seul public qui prévaut toutefois, et auquel le chercheur est invité à s'adresser, est celui que forment ses collègues. Au nom de l'idéal habermassien d'une «discussion rationnelle », l'échange légitime se réduit à celui d'une conversation entre savants, parfaitement neutre et séparée de toutes considérations sociétales ou politiques. Ainsi, la progressive disparition de la « forme-livre » au bénéfice de la « formearticle » des publications, ce dont rendent bien compte l'évolution des coûts des bibliothèques universitaires ou les modes d'évaluation des chercheurs, signale un tel glissement. Les articles dits « scientifiques » sont réputés un élément plus fiable pour apprécier le sérieux et la rigueur du spécialiste. Mais le risque d'une telle tournure n'est-elle pas toutefois de consacrer des discussions sans fins sur des sujets qui n'intéressent personne? N'y a t-il pas danger pour la « logique de la création » elle-même, lorsqu'on prive ainsi la recherche à produire des circulations inédites entre savants et profanes, comme si le chercheur devenait assigné à résidence universitaire?

A l'aune de ces trois considérations, le mouvement actuel de réforme de l'Université et de la recherche ressemble donc fort à une mauvaise aventure. La désaffection progressive chez les étudiants à l'égard du monde universitaire, le faible intérêt que rencontrent les publications académiques voire la baisse du niveau général des débats théoriques que Lagasnerie constate à l'heure actuelle, au regard de la période plus faste qui l'a précédée, pourraient, certes, être lus comme le résultat de facteurs externes comme la baisse de consommation de biens culturels, la pression du marché dans le secteur éditorial au seul bénéfice de la recherche de rentabilité à court terme, l'emprise croissante du champ médiatique sur la vie des idées, voire la dégradation du niveau d'exigence des journalistes eux-mêmes. Mais c'est la responsabilité de l'Université, et ce qu'elle fait à la pensée, que l'auteur interroge frontalement. 
Assurément, nous l'aurons compris, l'ouvrage que consacre Geoffroy de Lagasnerie au monde intellectuel se veut éminemment critique. C'est un livre inquiet, avisé quand il pointe les dérives de la politique actuelle de «professionnalisation» des savoirs censée garantir l'indépendance et l'autonomie nécessaires à la vie intellectuelle pour pouvoir innover, alors même que les parcours des chercheurs étudiés par l'auteur indiquent l'inverse. La publication récente de Luc Boltanski, que ce dernier consacre notamment à l'histoire de la mise en place des Actes de la recherche en sciences sociales en 1975, peut ici fournir d'utiles compléments (1). L'ouvrage de Lagasnerie est également un livre qu'on a envie de suivre, par le seul fait qu'il situe la logique de la création sur le double plan de la jubilation proprement intellectuelle et des institutions qui en permettent l'épanouissement. Le propos se veut incisif, et c'est tant mieux, puisque c'est une révolution conservatrice à l'œuvre depuis les années 80 que l'auteur décrit, qui tend à fragiliser cette capacité collective d'imagination créatrice. Mais c'est aussi un livre profondément nostalgique - et c'est là sûrement, à nos yeux, ce qui fait qu'on ne peut en partager vraiment la totalité de l'analyse. En fait, Lagasnerie nous paraît nostalgique par deux fois.

Une première fois, parce que le monde de la recherche d'avant les années 80 semble celui d'un temps exagérément béni. C'est celui des grandes idées, des livres intelligents, des débats théoriques puissants, des engagements politiques courageux, quand aujourd'hui triompherait une relative médiocrité. Il est permis d'en douter, d'autant que l'ouvrage ne donne finalement que peu d'exemples concrets en mesure d'étayer ces considérations : n'y a t-il pas, depuis la période dorée qui fait l'objet de son attention, des renouvellements théoriques tout aussi notables (2) ? Des lieux hybrides de production de la recherche, quoiqu'on en pense, n'ont-ils pas été inventés depuis (3) ? N'y a t-il pas des lieux de débats intellectuels qui ont pu émerger, quand bien même ils prendraient des formes différentes de celles de la « revue » ou de centres

1.- Voir Boltanski, Luc, Rendre la réalité inacceptable, à propos de la production de l'idéologie dominante, Paris, Demopolis, coll. Essai, 2008

2.- Pour un aperçu de ce renouvellement théorique dans les sciences humaines et sociales, voir par exemple François Dosse, L'empire du sens, La Découverte, Paris, 1995

3.- Des formes contractuelles de la recherche en partenariat à l'essor des conventions CIFRE en matière de production de thèses ne révèlent-ils pas des déplacements possibles de la relation du chercheur à son terrain et des manières d'intégrer les indigènes dans la production même de la recherche ? Des projets d'anti-disciplinarité n'ont-ils pas été à l'origine de la création de la majorité des laboratoires de sciences sociales des grandes écoles d'ingénieurs, sources d'innovations théoriques en sociologie, en gestion, en économie, en philosophie ou en histoire parfaitement récentes, quand bien même la politique de la recherche dénoncée par l'auteur pourrait les fragiliser? 
d'enseignement (4) ? Dans quelles mesures l'opposition entre les années 60 et 70 d'un côté et la période actuelle de l'autre, ne risque t-elle pas de devenir caricaturale, empêchant de distinguer des « logiques de création » possiblement différentes selon les auteurs analysés, y compris dans leur rapport aux institutions (5). Est-il bien certain que les débats experts d'aujourd'hui sur la méthodologie ou les concepts pointus des chercheurs soient terriblement moins utiles et plus ennuyeux au regard des querelles politiques plus ardentes qui les ont précédés?

Mais de manière plus centrale selon nous, La logique de la création apparaît nostalgique une seconde fois. En opposant à la rhétorique convenue de «l'autonomie » la perspective plus joyeuse de «l'hétéronomie », à la position du «professionnel» compétent sur un domaine limité la figure romantique de l'«intellectuel» qui s'adresse à la société, aux débats « techniques » mais qui tournent à vide la « repolitisation » de l'espace théorique en lien avec les questions du monde, l'on pourra se demander si, finalement, ce n'est pas une même épistémologie de l'activité scientifique qui est activée par l'auteur que celle qui est à l'œuvre dans les réformes actuelles. Une épistémologie un peu datée, disons-le, qui consiste à naturaliser les oppositions hétéronomie/autonomie, sciences/société ou technique/politique plutôt que de les voir comme le produit d'un travail, le résultat d'un processus de lente fabrication qui font de ces oppositions, non des points de départ, mais des points d'arrivée. Les analyses récentes de la nouvelle sociologie des sciences, qui ont contribué à réexaminer le contenu et les enjeux de ces tensions, et qui ont conduit à s'interroger sur la place du politique ou des profanes dans la dynamique de la recherche, auraient très certainement gagné à être discutées (6). N'est-ce pas aussi à cet endroit précis du travail de recherche que se déploie, dans sa vérité, la créativité scientifique?

Bref, l'ouvrage de Geoffroy de Lagasnerie est un livre qu'on aborde au départ avec une indéniable réjouissance. Les propos sont vifs, les questions posées sont pertinentes. Mais au fur et à mesure que l'on progresse dans la lecture, l'analyse - au-delà des simplifications inévitables à l'exercice de genre, mais qui peuvent agacer - finit par laisser entrevoir une

4.- La considération de l'internet sur la vie de la recherche, pourtant décisif dans les pratiques de lecture, d'échanges et d'évaluation, apparaît curieusement absente de l'ouvrage

5.- On pense, par exemple, à la stratégie de Bourdieu à constituer une «école » sociologique, aux antipodes de celle d'un Deleuze à l'origine d'un «mouvement», mais jamais d'une école avec ses disciples et ses épigones. La circulation des concepts des uns et des autres dépend aussi très largement de ces stratégies.

6.- Voir par exemple Latour, Bruno, Changer de société, Refaire de la sociologie, La découverte, Paris, 2006 ou les travaux d'Isabelle Stengers, Cosmopolitique, coll. Essais, La découverte, Paris, 2003 
même conception de l'activité de recherche, fondée sur les mêmes implicites, les mêmes mythes que celle que l'auteur espère combattre. Les thèses développées par l'auteur sur la créativité intellectuelle, ses espaces nécessaires de liberté et ses publics demeurent parfaitement éclairantes. Mais elles le conduisent à des recommandations peu convaincantes. On comprend ce qu'elles combattent. Mais on s'interroge sur leur portée, aussi tranchantes que ces poignards dont se munissent parfois certains personnages de Tintin et Milou pour assaillir un ennemi et dont ils s'aperçoivent, après en avoir brandi la lame, qu'elle est en fer blanc. La logique de la création n'en a pas moins un immense mérite : elle ne laisse pas indifférent. 